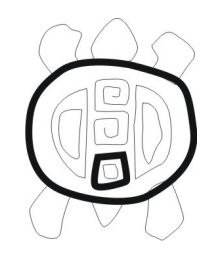

RMA

Dossier - Arqueología

\title{
Explorando la variabilidad morfométrica del conjunto de camélidos pequeños durante el Arcaico Tardío y el Formativo Temprano en Quebrada Tulán, norte de Chile
}

Isabel Cartajena Fasting

Departamento de Antropología, Universidad de Chile E-mail: icartaje@uchile.cl

\begin{abstract}
Resumen
Frecuentemente en el registro arqueofaunístico de sitios arqueológicos ubicados en la vertiente occidental de la puna de Atacama, es posible diferenciar un conjunto de camélidos grandes y otro de menor tamaño. En general, el grupo de camélidos grandes ha recibido mayor atención, existiendo un escaso conocimiento del grupo de tamaño más pequeño. En este trabajo se explora el conjunto de camélidos pequeños a través de la utilización de métodos osteométricos. Por una parte, se consideran colecciones actuales de alpacas y vicuñas en las que se analizan las distintas variables que pudiesen estar incidiendo en la variabilidad de los conjuntos. Se otorga especial énfasis a la diferenciación entre falanges anteriores y posteriores, variación interespecifica y dimorfismo sexual. Por otra parte, se analizan las primeras falanges de los sitios Tulán-52 (Arcaico Tardío ca. 4500-3800 A.P.) y Tulán-54 (Formativo Temprano ca. 3200-2300 A.P.) con el fin de caracterizar la variabilidad que presentan los conjuntos a través del tiempo, mediante la comparación de los resultados del análisis de muestras actuales y arqueológicas. Ambos conjuntos arqueofaunisticos son muy similares a pesar de las diferencias temporales. Los especimenes analizados corresponderían a vicuñas, lo que denotaría la importancia de la caza de la vicuña a través del tiempo.
\end{abstract}

Palabras clave: arqueofaunas, osteometria, alpacas, vicuñas.

Exploring the morphometric variability of lesser camelids from Quebrada Tulán (northern Chile) during the Late Archaic and Early Formative.

\begin{abstract}
Frequently, it is possible to differentiate among large and small size camelids when analysing registers from archaeological sites located at the occidental slope of Puna de Atacama. However, larger animals have received most of the attention having less knowledge about the group of small size camelids. In this paper and by using osteometric methods we study this small size later group. On one hand, we analyse different variables that may affect variability among modern alpacas and vicuñas collections. We focus our work on differences among anterior and posterior phalanxes, inter specific variation and sexual dimorphism. On the other hand, we analyse first phalanxes from Tulán-52 (Late Archaic ca. 4500-3800 BP) and Tulán-54 (Early Formative ca. 3200-2300 BP) characterising their variability through time by comparing current and archaeological samples. Both archaeofaunal assemblages are similar despite their temporal distance. The analyzed specimens correspond to vicuñas denoting the importance of vicuña hunting through time
\end{abstract}

Keywords: archaeofauna, osteometry, alpacas, vicuñas.

En la vertiente occidental de la puna de Atacama se generó un conjunto de condiciones culturales, sociales y ambientales favorables que permitió la transición de las sociedades cazadoras-recolectaras del Arcaico Tardío (ca. 5000-3800 AP) a las sociedades de carácter pastoralista del Formativo Temprano (ca. 3200-2400 AP). Este cambio se encuentra marcado, entre otros aspectos, por la domesticación de los camélidos, una disminución de la movilidad residencial, una mayor aglomeración poblacional, la producción de bienes excedentarios, redes de interacción macroespacial y una creciente complejidad ritual (Núñez et al. 2006). En este contexto, en nuestra área de estudio, la fase Tilocalar puede definirse a partir de un conjunto de indicadores culturales que representan el inicio del Formativo, el surgimiento de la vida aldeana, la intensificación y ampliación de diversas prácticas productivas, un fuerte énfasis en las manifestaciones rituales y los inicios de la tradición pastoralista atacameña, aunque aún se aprecia un fuerte énfasis en la caza (Núñez et al. 2006).

Durante este período, la llama habría sido domesticada en diversos lugares de los Andes, entre ellos el área circumpuneña. La utilización de métodos osteométricos ha permitido reconocer una alta variabilidad métrica dentro de los conjuntos, advirtiéndose por una parte la presencia de dos grupos, uno de tamaño grande (guanaco/llama) 
y otro de tamaño pequeño (vicuña/alpaca). Asimismo, también en el interior de cada uno de ellos ha sido posible reconocer diversos grupos de tamaño. En nuestra región de estudio, la atención se ha centrado principalmente en el conjunto de camélidos de mayor tamaño, donde las variaciones han sido interpretadas como resultado de la domesticación de los guanacos y la incipiente crianza de las llamas (Cartajena et al. 2007, Hesse 1982, Mengoni 2008, Mengoni y Yacobaccio 2006, Yacobaccio 2003). Es por esta razón que, a pesar de que el conjunto de camélidos pequeños se registra en gran parte de los sitios, ha recibido menor atención por parte de los investigadores, por lo cual se trata de un conjunto escasamente estudiado. Esta falta de interés también obedece a que, contrariamente al caso de la llama, la domesticación de la alpaca se habría producido solamente en los Andes Centrales. A través de la morfología de los incisivos, Wheeler (1984) logró identificar algunos especímenes del "tipo alpaca" en yacimientos arcaicos de la Puna Central de Perú (Junín), los que corresponderían a las evidencias más tempranas de la domesticación de la alpaca entre los 6.000-5.500 A.P. (Wheeler 1995). Recientes estudios moleculares sugieren una gran similitud genética entre la alpaca y la vicuña y entre la llama y el guanaco (Kadwell et al. 2001, Marín et al. 2007), por lo que en la actualidad prevalecería un modelo basado principalmente en la evidencia arqueozoológica y genética, donde la llama habría sido domesticada a partir del guanaco y la alpaca de la vicuña, con hibridaciones posteriores (Marín et al. 2007). En los Andes Centro-Sur, la alpaca habría ido adquiriendo importancia durante el Formativo entre los 3570 y 3000 AP, mientras la vicuña comenzaría a ser reemplazada por un camélido más grande (Mengoni 2008).

No obstante, en nuestra área de estudio, un análisis secuencial de los restos arqueofaunísticos desde el Arcaico Temprano hasta el Formativo Temprano (ca. 9.000 A.P. hasta 2.400 A.P) sugiere que el conjunto de camélidos pequeños presenta menores variaciones que el conjunto de camélidos grandes a través del tiempo. Lo anterior señala una escasa incidencia de factores ambientales y la ausencia de las variaciones que en el caso del conjunto de los camélidos grandes, se atribuiría al proceso de domesticación y una progresiva consolidación de la tenencia de rebaños (Cartajena et al. 2007). No obstante, por una parte, en el caso del sitio formativo Tulán-54, se registraron tres incisivos con una morfología similar a la alpaca (Cartajena et al. 2009); por otra, en la cuenca del Loa Medio (ubicada más al norte) en el sitio formativo Chiu-Chiu 273, se registraron principalmente extremidades inferiores de camélidos y cráneos en contextos fúnebres. De acuerdo con los estándares ocupados en esa oportunidad (Kent 1982), las extremidades de camélidos pequeños correspondían al rango de las alpacas actuales, sugiriendo la presencia de ésta. Sin embargo, puesto que también se encontraron incisivos de vicuñas, las mediadas se podrían estar superponiendo con estas últimas (Cartajena y Concha 1997). De acuerdo con las evidencias disponibles hasta la fecha, la presencia de alpaca en los registros de esta época no es concluyente.

Finalmente, la dificultad de reconocer el impacto de la variación regional en el tamaño de los camélidos, ha sido un impedimento en los análisis osteométricos, especialmente al utilizar colecciones de referencia actuales (Mengoni y Yacobaccio 2006). En este contexto, los objetivos del presente trabajo son (a) identificar los factores de variabilidad en el conjunto de camélidos pequeños (alpacas y vicuñas); y (b) caracterizar la composición taxonómica de los conjuntos de camélidos pequeños provenientes de los sitios Tulán-52 y 54. A través del estudio de colecciones de referencia actuales se busca interpretar las variaciones que pudieran presentar los conjuntos faunísticos de los sitios a través del tiempo.

\section{Materiales y métodos}

Los restos de camélidos analizados en este trabajo provienen de los sitios arqueológicos Tulán-52 y Tulán-54, ubicados Quebrada Tulán, al sureste del Salar de Atacama. La quebrada tiene una extensión de $30 \mathrm{~km}$ a través de los cuales es posible conectar el borde oriental del Salar de Atacama (2.300-2.380 msnm), los oasis pedemontanos (2.380-2.750 msnm), la zona de quebradas intermedias donde se ubican los yacimientos (ca. $2960 \mathrm{msnm}$ ) con las tierras altas (sobre los 3.500 msnm) (Núñez y Santoro 1988). Aquí se concentran recursos de agua subterránea y superficial, forraje estable, canteras y pisos ecológicos complementarios, favoreciendo el desarrollo de asentamientos estables y circunscriptos (Núñez et al. 2006). Durante las ocupaciones arcaicas habrían prevalecido condiciones más áridas, las que sin embargo, en un contexto de aridez regional habrían sido favorables para los asentamientos arcaicos. Alrededor de los 3600 AP, se observa un incremento en la humedad, estableciéndose las condiciones actuales a los ca. 3000 AP, coincidente con las ocupaciones formativas tempranas (Grosjean et al. 2001, 2005).

El asentamiento arcaico Tulán-52 se caracteriza por la presencia de estructuras circulares y subcirculares aglomeradas, construidas con grandes bloques verticales, con dataciones entre los 4580 a 3860 A.P. Las estructuras se encuentran completamente cubiertas por depósitos estratificados compuestos por fogones, restos óseos, líticos y vegetales. Las materias primas más frecuentes corresponden a rocas locales silíceas, basaltos y tobas, aunque existe una importante presencia de otras de origen foráneo como la obsidiana, la que indica cierta movilidad hacia las tierras altas. Por otra parte, los restos malacológicos del Pacífico (Oliva peruviana, Pecten purpuratus) señalan la existencia de contactos hacia la costa (Núñez et al. 2006). Los análisis arqueofaunísticos en Tulán-52 permitieron identificar durante el Arcaico Tardío la presencia de dos grupos de tamaño, uno pequeño (vicuña) y otro grande (guanaco/llama). Este último presenta una gran variabilidad métrica, lo que indicaría la presencia de los primeros animales domésticos (Cartajena et al. 2007). La 
presencia de animales domésticos también se observó a través del análisis de fanéreos, donde predomina la vicuña, seguida por guanaco, y una escasa presencia de fanéreos de llamas (Benavente 2005-2006).

Tulán-54 corresponde a un asentamiento formativo temprano emplazado en el borde sur de la quebrada de Tulán, a unos $300 \mathrm{~m}$ al oeste del asentamiento arcaico Tulán-52. Hasta ahora se han identificado varios sectores que muestran una compleja organización del espacio, destacando un gran templete central y otras estructuras de probable carácter habitacional a su alrededor, datados entre los 2380 a 3080 A.P. Bajo las capas de un montículo estratificado, se registró una estructura ovalada compuesta por un muro perimetral construido con grandes bloques verticales, con nichos rectangulares a nivel del piso. En el interior se encuentran divisiones internas y una estructura central. En el piso se registran las inhumaciones de 24 neonatos humanos, los que fueron dispuestos en pozos apegados al muro perimetral y también al interior de la estructura central (Núñez et al. 2006). Los análisis arqueofaunísticos permitieron segregar nuevamente dos grupos de tamaño, no obstante, el conjunto de tamaño grande tiene una menor dispersión, lo que podría deberse a la maduración del proceso de domesticación, como también a la selección de un grupo más homogéneo para actividades doméstico-rituales propias del templete de Tulán-54. Como se mencionó anteriormente, se registraron además tres incisivos con características propias de la alpaca (Cartajena et al. 2009). Los fanéreos permitieron determinar nuevamente, la presencia mayoritaria de fibras y vellones de vicuña, seguidas por guanaco y en menor frecuencia de llama, junto a una posible fibra de alpaca (Benavente 2005-2006, Cartajena et al. 2009).

A pesar de los esfuerzos realizados por diversos autores, aún no existe un consenso en la metodología para diferenciar, con absoluta certeza, las cuatro formas de camélidos sudamericanos en función de los restos óseos recuperados (Bonavía 1996:109). Algunas de las herramientas metodológicas utilizadas para la determinación taxonómica han sido las medidas osteológicas. Sin embargo, aunque se pueden separar con relativa facilidad los restos de camélidos en dos grupos según su tamaño, dentro de cada uno de estos grupos se encuentra una especie silvestre y una forma doméstica. Las técnicas osteométricas aplicadas a los camélidos actuales han sido sujeto de un amplio debate desde comienzos del siglo pasado (Marelli 1912, Lönnberg 1913, León 1933, entre otros, en Herre 1952).

Los trabajos referidos a restos de animales provenientes de sitios arqueológicos con la aplicación de métodos métricos, comienzan en el área andina con los trabajos de Wing (1972). Estos son continuados por diversos autores, entre ellos Kent (1982) quien refina la metodología estadística mediante el uso de funciones discriminantes lineales para separar las cuatro especies considerando diversas medidas. Posteriormente, Moore (1989) plantea la dificultad para asignar restos de camélidos provenientes de sitios arqueológicos a alguno de los grupos de animales actuales y a través técnicas gráficas y estadísticas simples describe el cambio de ambos grupos de tamaño (grandes y pequeños) en el tiempo. En el Noroeste Argentino, la implementación de métodos osteométricos ha sido utilizada por diversos autores (Elkin et al. 1991, Izeta 2004, 2007, Mengoni 2008, Yacobaccio et al. 1997-1998, entre otros). Contrariamente, en nuestra área de estudio la osteometría ha sido poco utilizada (Hesse 1982, Cartajena y Concha 1997, Cartajena et al. 2007).

Sin embargo, los problemas de asignación taxonómica a través de medidas osteológicas persisten debido principalmente a la falta de diferencias significativas de tamaño entre los animales domésticos y silvestres (llama/ guanaco y alpaca/vicuña), por lo que los métodos métricos en general se limitan a la separación entre grandes y pequeños (Wheeler 1991). No obstante, el desarrollo y utilización de técnicas de análisis multivariado aplicadas en otras regiones ha permitido la separación entre taxones que presentan estrecha similitud (Menegaz et al. 1988, Menegaz et al. 1989), las que también han sido aplicadas en el noroeste argentino (Izeta y Cortés 2006)

Generalmente, se han utilizado medidas osteométricas provenientes de colecciones de referencia actuales, las que son llevadas a gráficos de dispersión con el fin de observar la distribución de las medidas de los conjuntos arqueofaunísticos y así poder asignarlas a los diversos taxones (Miller y Gill 1990, Miller y Burger 1995). Sin embargo, una de las críticas frecuentes a la aplicación de modelos basados en las dimensiones de especies modernas, radica en que se ignora el largo período de selección y cambio que pueden no ser reconocidos a través de los estándares actuales (Moore 1989:317).

A pesar de los problemas y las limitantes antes señaladas, en este trabajo se propone explorar la variabilidad de los conjuntos a través de estudios osteométricos. Con este fin se utilizan dos medidas correspondientes al ancho máximo de la superficie articular proximal (BFp) y la profundidad máxima (Dp) de la primera falange de acuerdo a los estándares propuestos por von den Driesch (1999). La elección de estas variables se debe a la abundancia de este elemento en el registro, la posibilidad de obtener medidas estandarizadas que pueden ser comparadas y controlar por ejemplo factores que pudieran estar incidiendo en el tamaño como la edad. Finalmente, éstas presentan una buena preservación en los sitios arqueológicos (Miller y Burger 1995, Kent 1982).

Se consideraron métodos de análisis uni, bi y multivariados. Para el caso de los primeros se utilizan herramientas estadísticas descriptivas como las pruebas de diferencias de media, las que buscan determinar si existen diferencias estadísticamente significativas entre dos grupos de medidas (López 2001), para lo cual se utilizó el software STATA 10.0. En el caso de los análisis bivariados se utili- 
zan los gráficos de dispersión, ya que como se mencionó anteriormente, esta herramienta permite asignar a un espécimen a un grupo en particular, a través de la distribución de pares de medidas en un plano cartesiano. Por su parte, el análisis de componentes principales permite separar vectorialmente grupos de medidas de manera de minimizar las diferencias entre los miembros de un mismo grupo y maximizar las diferencias entre grupos (López 2001), utilizándose en este caso software SPSS 12.0. Mediante estos análisis es posible segregar las muestras por forma y tamaño a la vez que permite compararlas con categorías taxonómicas predeterminadas, en este caso particular vicuña y alpaca.

En este trabajo se exploran dos vertientes. La primera, referida a muestras actuales en las que se analizan las distintas variables que pudiesen estar incidiendo en la variabilidad de los conjuntos. Se otorga especial énfasis a la diferenciación entre falanges anteriores y posteriores, variación interespecífica y dimorfismo sexual. Con este fin se trabajó con esqueletos de referencia actuales de sexo y edad conocidos correspondientes a alpacas y vicuñas (Anexo 1). En relación a las muestras arqueofaunísticas, se busca explorar la variabilidad de los conjuntos a través del tiempo y determinar si dicha variabilidad pudiese estar relacionada con las variables antes mencionadas.

\section{Diferenciación de falanges anteriores y posteriores}

Las primeras falanges fueron separadas entre anteriores y posteriores a partir de rasgos morfológicos, los que se presentan con mayor claridad en el conjunto de camélidos de tamaño grande (guanacos y llamas) que en camélidos pequeños (alpacas y vicuñas). La primera falange anterior es más larga que la posterior, diferencia que es nuevamente más evidente para los camélidos grandes que para los más pequeños. Al considerar el largo máximo (GL, von den Driesch 1999) en los animales actuales, la diferencia en la longitud de las falanges anteriores y posteriores, expresadas en términos porcentuales es de un 7 a $11 \%$ en alpacas y entre un 7 y $14 \%$ en el caso de las vicuñas (Cartajena 2002). Estos porcentajes son mayores a los señalados por Moore (1989:325) quien señala una diferencia entre la longitud de las falanges anteriores y posteriores de vicuñas de 1,5 a 6,8\%. Dadas las características de la muestra arqueofaunística, en este trabajo se hizo especial hincapié en la morfología de la epífisis proximal, especialmente en la forma de la superficie articular y las superficies de inserción muscular en la cara palmar y plantar.

La superficie articular proximal de la primera falange anterior presenta una forma más cuadrangular. La superficie articular proximal de la falange posterior se estira más en el sentido del eje axial-abaxial, con un borde abaxial marcadamente biselado (Cartajena 2002) (ver Anexo 2e, indicador D).

Las superficies de inserción muscular en la cara palmar (tuberositas flexoria) de la primera falange anterior, son más largas y se extienden hacia distal, más desarrolladas y se encuentran muy bien delimitadas. Las superficies de inserción muscular plantares en las primeras falanges posteriores son más cortas y menos delimitadas. No obstante, estas diferencias entre anteriores y posteriores se observan más claramente en los camélidos grandes y son menores (a veces casi inexistentes) en los pequeños (Cartajena 2002) (ver Anexo 2 b y d, indicador A).

\section{Diferenciación interespecífica}

En general, si bien se ha podido separar el conjunto de camélidos pequeños del conjunto de camélidos de tamaño grande, la separación taxonómica dentro de cada uno de ellos no ha sido fácil a raíz del grado de superposición que estas presentan. En este caso se llevaron las medidas a un gráfico de dispersión, lo que al igual que en el análisis de componente principal permite observar dos variables en conjunto, el primero de ellos a través de un plano cartesiano y el segundo a través del cruce de los dos factores principales de las variables seleccionadas (López 2001).

Los resultados obtenidos fueron cotejados con otras colecciones de referencia actuales con el fin de observar si el conjunto analizado en este trabajo presenta grandes diferencias, en especial referidas al tamaño de animales estudiados.

\section{Diferenciación por sexo}

Puesto que los animales actuales eran de sexo conocido, las primeras falanges de alpacas y vicuñas fueron diferenciadas por sexo con el fin de observar, a pesar de que los camélidos presentan un bajo dimorfismo sexual, si este se expresa métricamente tanto en el interior de cada uno de los taxones como entre ellos. Cabe señalar que en este caso el macho en las vicuñas es un poco más grande que la hembra, hecho que también se observa en las alpacas, donde entre los cuatro y cinco años el macho presenta un tamaño mayor que la hembra (Moore 1989).

\section{Resultados}

A continuación se entregan los resultados del análisis de las colecciones actuales, con el fin de contrastarlos con las muestras arqueológicas provenientes de los sitios Tulán-52 y 54.

\section{Diferenciación de falanges anteriores y posteriores en alpacas y vicuñas actuales}

Como se mencionó anteriormente, se analizaron las primeras falanges anteriores y posteriores de las colecciones actuales de vicuñas y alpacas. Una vez separadas, las medidas obtenidas para la epífisis proximal (BFp y Dp) fueron llevadas a un gráfico de dispersión (Figura 1).

Como se puede observar en el gráfico, las medidas se 
agrupan en dos conjuntos fácilmente diferenciables, uno correspondiente a las vicuñas y el otro a las alpacas. Es interesante observar que sólo en dos casos se observa una superposición entre las medidas de alpacas y vicuñas, especialmente entre las falanges posteriores de alpaca y el conjunto de vicuñas. Dentro del conjunto de las alpacas se observa una clara separación entre anteriores y posteriores, lo que en el conjunto de vicuñas es más difuso.

Al analizar las variables BFp y Dp por separado (Figuras 2 y
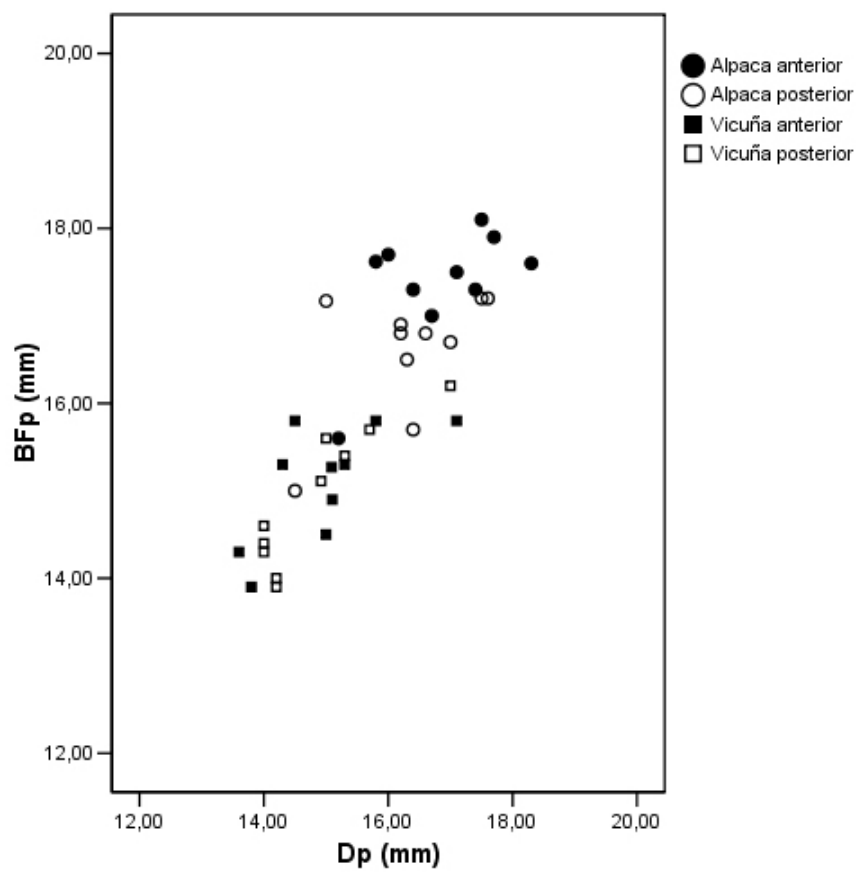

Figura 1: Medidas del ancho máximo de la superficie articular proximal (BFp) y de la profundidad máxima (Dp) de las primeras falanges de vicuñas y alpacas actuales.

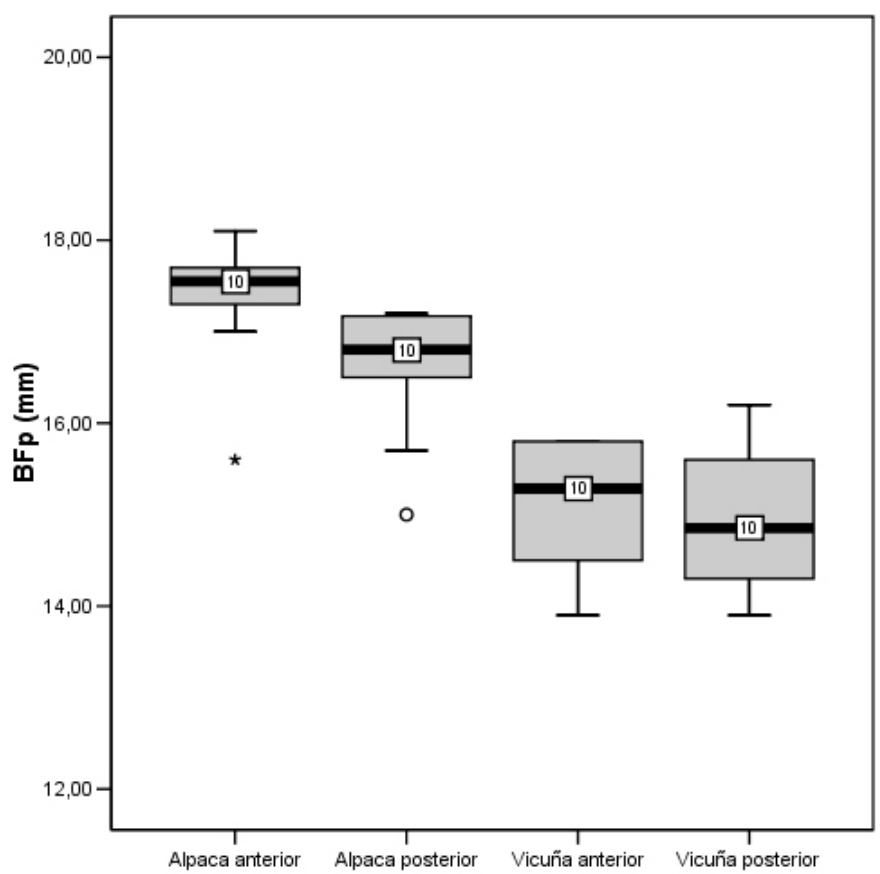

Figura 2: Medidas del ancho máximo de la superficie articular proximal (BFp) de las primeras falanges de vicuñas y alpacas actuales.
3), se puede observar que la primera de ellas permite una mejor separación dentro del conjunto de alpacas, hecho que también se observa en la profundidad máxima de la epífisis proximal Las pruebas de diferencia de medias realizadas sugieren, al igual que los gráficos, que BFp permite una mejor separación de las falanges anteriores y posteriores en el conjunto de alpacas, no presentando Dp resultados significativos para la separación de las falanges dentro de cada uno de los conjuntos.

Es importante señalar que BFp y Dp también resultaron significativas para la separación de falanges posteriores

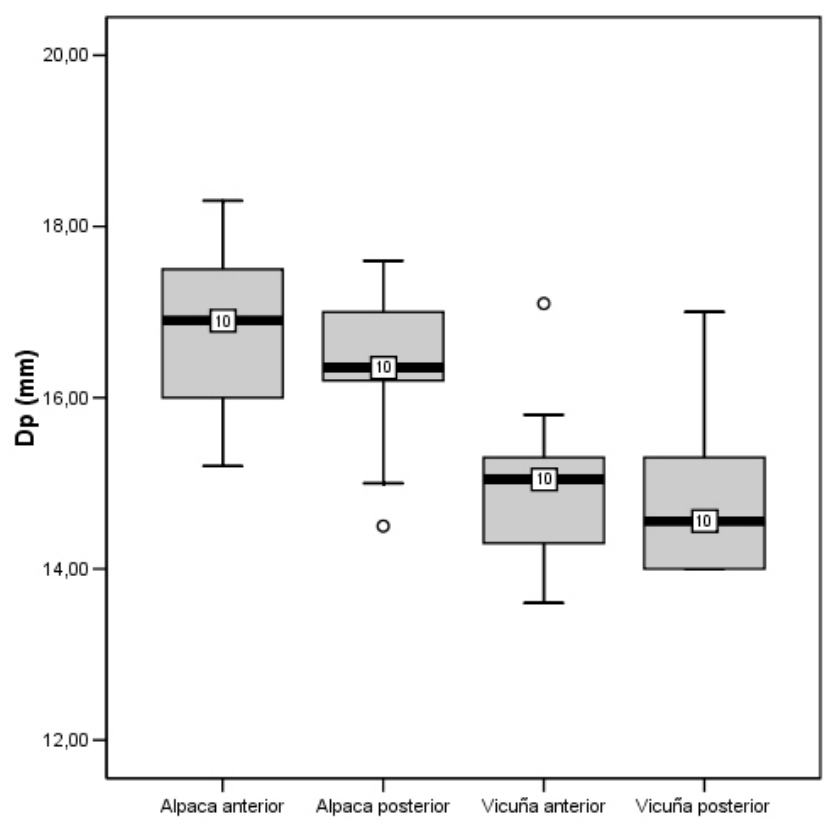

Figura 3: Medidas de la profundidad máxima (Dp) de la primeras falanges de vicuñas y alpacas actuales.

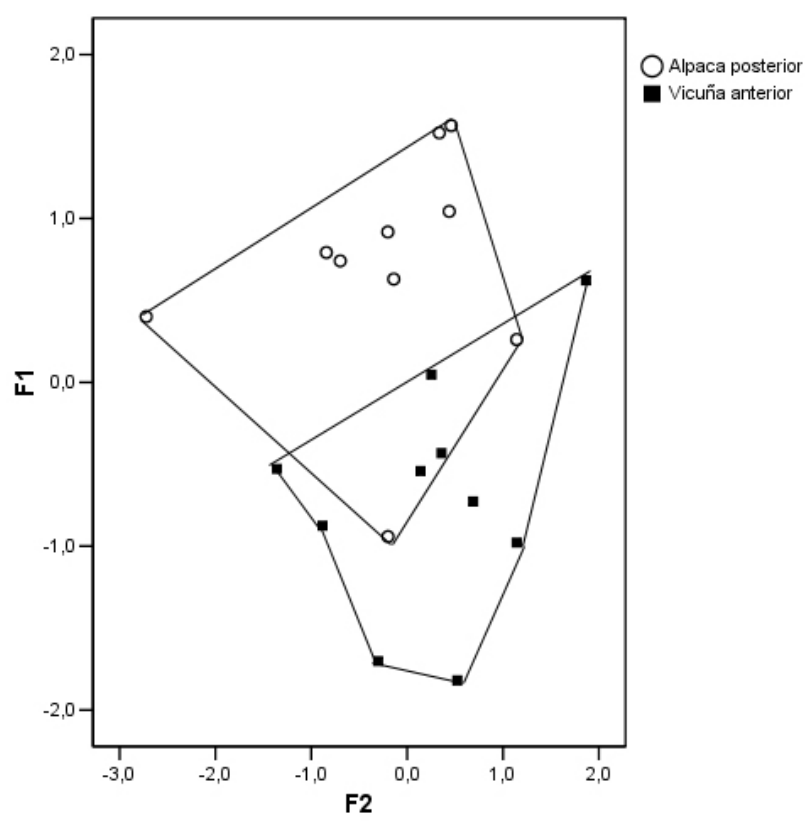

Figura 4: Análisis de componentes principales para dos variables (BFp y DP) para las primeras falanges anteriores de vicuñas y posteriores de alpacas. 
de alpaca y anteriores de vicuñas, donde en general se da la mayor superposición entre los conjuntos (Anexo 3). A través de un análisis de componentes principales para ambas variables se observa una situación similar a la antes descripta (Figura 4).

\section{Diferenciación interespecífica entre alpacas y vicuñas} actuales

Al considerar los dos taxones en conjunto, pero ahora se-

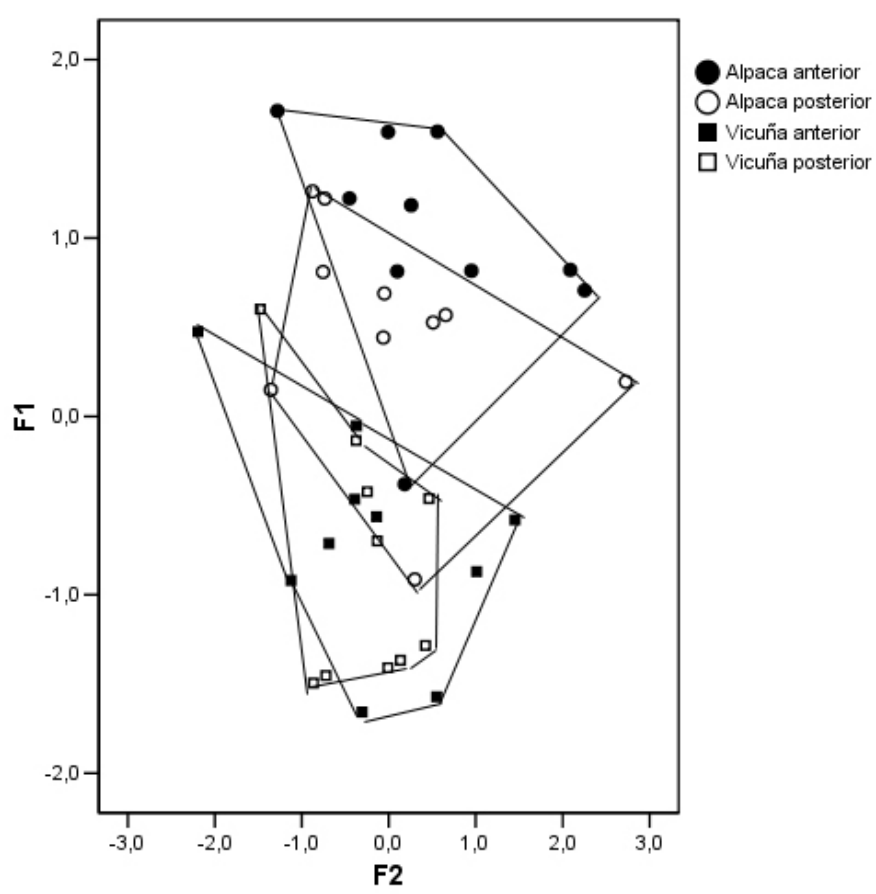

Figura 5: Análisis de componentes principales para dos variables (BFp y DP) para las primeras falanges anteriores y posteriores de vicuñas y alpacas.

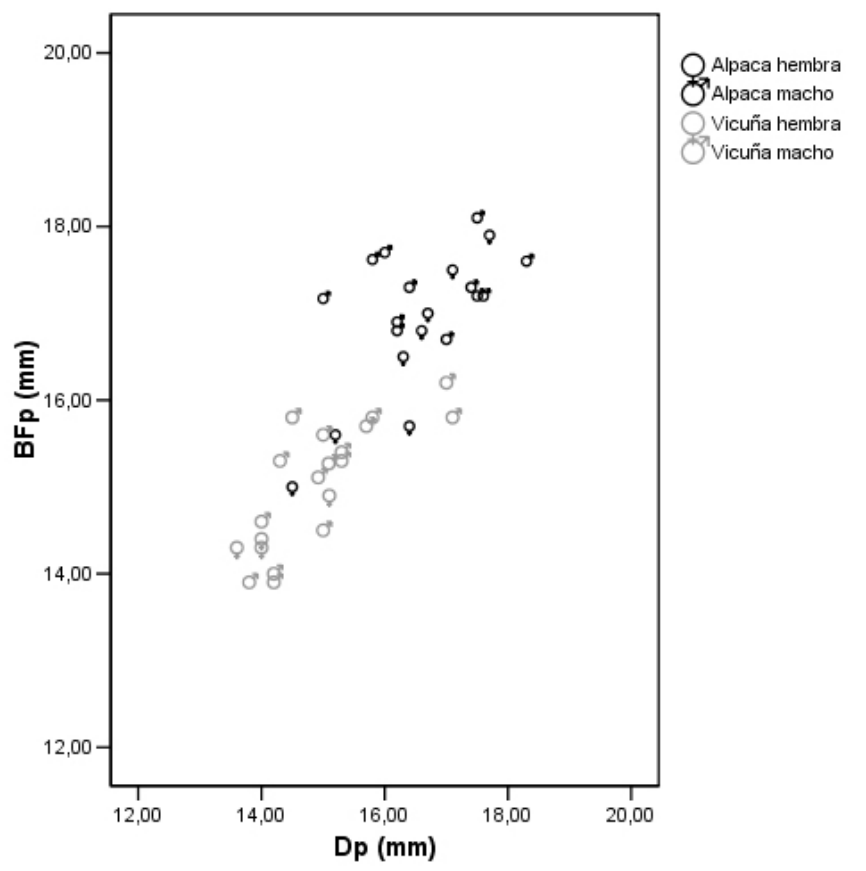

Figura 6: Medidas del ancho máximo de la superficie articular proximal (BFp) y profundidad máxima (Dp) de las primeras falanges de vicuñas y alpacas actuales separadas por sexo. parando las falanges anteriores y posteriores, el resultado obtenido del análisis de componentes principales permite observar una separación entre alpacas y vicuñas, con las superposiciones antes mencionadas (Figura 5).

Con el fin de evaluar diferencias métricas con animales provenientes de otras colecciones de referencia, se compararon las medidas publicadas por otros autores (Tabla 1). En general, a pesar de la diversa procedencia de los animales las medidas son similares, observándose una superposición mínima entre el rango de las alpacas y las vicuñas.

La mayoría de las vicuñas medidas corresponderían a Vicugna vicugna mensalis, la que se distribuye en Perú, Bolivia y Chile entre los $9^{\circ}$ y $18^{\circ} \mathrm{S}$. Esta se caracteriza por ser una forma más grácil (con una alzada de $45 \mathrm{~cm}$ ). Más al sur entre los $18-29^{\circ} \mathrm{S}$ se encuentra Vicugna vicugna vicugna cuya distribución incluye Bolivia, Chile y Argentina y que a diferencia de la anterior por cuanto presenta una mayor alzada (Marin et al. 2007). Por tanto, en nuestro territorio de estudio cabría esperar medidas mayores a las utilizadas comúnmente como comparación.

Por su parte, la alpaca es la especie más pequeña de los camélidos domésticos y comparte muchas características morfolólogicas con las vicuñas más septentrionales, lo que sugiere que la alpaca fue domesticada a partir de poblaciones locales de V. v. mensalis (Marin et al. 2007). Las medias de las diversas alpacas utilizadas por Kent (1982) son similares a las utilizadas en este estudio, sin embargo, presentan mayor dispersión en el rango superior (Kent 1982).

\section{Diferenciación por sexo en alpacas y vicuñas actuales}

En cuanto al impacto del dimorfismo sexual en las medidas para las primeras falanges de alpacas y vicuñas, no se observa una separación clara dentro de cada grupo en términos de sexos, si bien parece haber una tendencia a que los machos presenten un mayor tamaño en cada uno de los conjuntos. Es interesante notar que el único caso de superposición entre grupos se observa entre las alpacas hembras y vicuñas macho (Figura 6). A pesar de esta situación, los resultados de las pruebas de diferencias de medias tanto para ancho máximo de la superficie articular proximal como para la profundidad máxima señalan que estadísticamente es posible separar las falanges de alpacas hembras y vicuñas machos (Anexo 3).

Si se consideran las falanges de vicuña en conjunto, es posible observar que las hembras son un poco más pequeñas que los machos tanto para las dimensiones de ancho máximo de la superficie articular proximal (BFp, Figura 7a) como para la profundidad máxima (Dp, Figura 7b). No obstante, las pruebas de diferencia de media realizados entre vicuñas machos y hembras no presentan resultados estadísticamente significativos que permitan su separación (Anexo 3). Si se incluye además la variable 
falange anterior-posterior, en el caso de las vicuñas es posible observar que las falanges se diferencian entre machos y hembras independientemente de que sean anteriores o posteriores. Sin embargo, es importante mencionar que sólo se contó con dos vicuñas hembras.

Las pruebas de diferencia de medias no presentaron resultados significativos para separar entre machos y hembras de alpacas, ya sea a través de BFp o Dp (Anexo 3). Sin embargo, tal como se puede observar en la Figura 7, cuando se incluye además de la variable sexo la variable posicional anterior-posterior, a diferencia de lo observado en las vicuñas, se aprecian diferencias, especialmente en relación al ancho máximo de la superficie articular proximal (BFp) donde en el caso de la falange anterior y de la posterior la hembra es más pequeña que el macho. Las falanges anteriores son más grandes en machos y hembras y las posteriores de hembras son las más pequeñas. A diferencia de las vicuñas, estas dos variables tomadas en conjunto aumentan la variabilidad para este taxón.

Los estudios realizados para el conjunto de alpacas y vicuñas actuales nos permiten generar las siguientes expectativas:

1. Las falanges anteriores y posteriores pueden ser separadas a través de criterios morfológicos. Esta separación es importante puesto que permite delimitar el área de superposición entre ambos taxones. Es importante señalar que salvo en un caso, la superposición se observa entre las falanges posteriores de las alpacas y las anteriores de las vicuñas, donde se encontró una falange anterior de alpaca en el conjunto de vicuñas. De acuerdo a lo anterior se puede señalar que conociendo la posición (anteriorposterior) en general, no se superponen las medidas de las falanges anteriores de alpacas con las anteriores de las vicuñas y ni tampoco las posteriores de alpaca con las posteriores de vicuña.
2. Para diferenciar entre taxones resultó discriminante la utilización de ambas variables (BFp y Dp), ya sea a través de gráficos bivariados o a través del análisis de componentes para dos variables. En el caso de utilizar una variable, si bien BFp y Dp resultaron significativas para la separación de ambos taxones en especial para el área de traslape entre alpacas traseras y vicuñas delanteras, BFp presenta mayor grado de robustez en la separación.

3. En relación con las variaciones que se pudiesen presentar debido al dimorfismo sexual, en el caso de las vicuñas, las falanges se diferencian en cuanto a la medida BFp y $\mathrm{Dp}$, donde independientemente de su posición anterior o posterior las vicuñas hembras son más pequeñas. En el caso de las alpacas, no sólo se observan diferencias entre falanges anteriores y posteriores, sino que al introducir la variable sexo es posible obtener una gradación en orden descendente entre falanges anteriores de machos, falanges anteriores de hembras, falanges posteriores de machos $y$, finalmente, falanges posteriores de hembras.

Los resultados antes señalados deberán ser contrastados en el futuro con una muestra más grande, con el fin de observar de mejor forma la variabilidad tanto intra como interespecífica. De igual forma, si bien se observan algunas diferencias en cuanto al tamaño en relación a otras colecciones de referencia, tanto las alpacas como las vicuñas actuales utilizadas en este trabajo se encuentran dentro de los rangos establecidos para las diversas colecciones.

\section{Análisis de las primeras falanges de los sitio Tulán-52 y 54}

Como se mencionó anteriormente, para este estudio se consideraron las primeras falanges provenientes de los sitios Tulán-52 y 54, específicamente aquellas que presentaban las medidas BFp y Dp. Estas fueron separadas en delanteras y traseras. Con el fin de establecer el

\begin{tabular}{|c|c|c|c|c|c|c|c|c|}
\hline $\begin{array}{c}\text { Colección de } \\
\text { referencia }\end{array}$ & $\begin{array}{l}\text { Primera falange } \\
\text { anterior/posterior }\end{array}$ & Medida & $n$ & $x$ & SD & Min. & Max. & Fuente \\
\hline V. vicugna & Anterior & BFp & 10 & 15,09 & 0,67 & 13,9 & 15,8 & Cartajena (2002, Anexo 4) \\
\hline V. vicugna & Anterior & FP1V2 & 7 & 15,53 & 0,25 & - & - & Kent (1982, Appendix IV.2) \\
\hline V. vicugna & Anterior & PHF2 & 9 & 16,68 & 1,32 & 14,3 & 17,9 & Izeta (2004: 410, Anexo 2) \\
\hline V. vicugna & Posterior & BFp & 10 & 14,92 & 0,79 & 13,9 & 16,2 & Cartajena (2002, Anexo 4) \\
\hline V. vicugna & Posterior & BP1V178 & 3 & 13,3 & 0,366 & - & - & Kent (1982, Appendix IV.2) \\
\hline V. vicugna & Posterior & PHF2 & 4 & 14,8 & 0,06 & 14,3 & 15,6 & Izeta (2004: 410, Anexo 2) \\
\hline V. vicugna & ant/post & latero-medial breadth ${ }^{1}$ & 16 & 15,5 & 0,87 & 15,0 & 16,0 & Miller y Burger (1995, Table 2) \\
\hline L. pacos & Anterior & BFp & 10 & 17,36 & 0,693 & 15,6 & 18,1 & Cartajena (2002, Anexo 4) \\
\hline L. pacos & Anterior & FP1V2 & 20 & 17,6 & 0,25 & - & - & Kent (1982, Appendix IV.2) \\
\hline L. pacos & Posterior & BFp & 10 & 16,6 & 0,715 & 15 & 17,2 & Cartajena (2002, Anexo 4) \\
\hline L. pacos & Posterior & BP1V178 & 21 & 16,9 & 0,248 & - & - & Kent (1982, Appendix IV.2) \\
\hline L. pacos & ant/post & latero-medial breadth ${ }^{1}$ & 55 & 17,2 & 1,28 & 16,8 & 17,5 & Miller y Burger (1995, Table 2) \\
\hline
\end{tabular}

Tabla 1: Medidas de vicuñas y alpacas actuales (ancho máximo de la superficie articular proximal de la primera falange) utilizadas como colecciones de referencia. ${ }^{1}$ De acuerdo a la Figura 6 (Miller y Burger 1995: 431) correspondería al ancho de la superficie articular. 


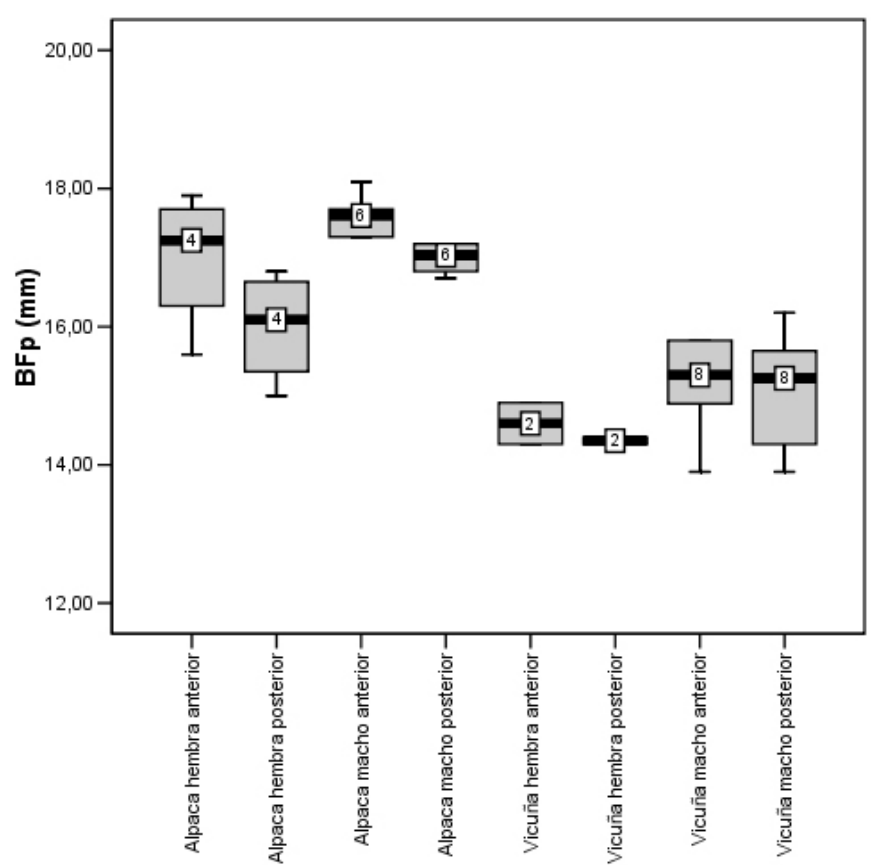

Figura 7a: Medidas del ancho máximo de la superficie articular proximal (BFp) de las primeras falanges de vicuñas y alpacas actuales separadas por sexo (macho-hembra) y posición (anterior-posterior).

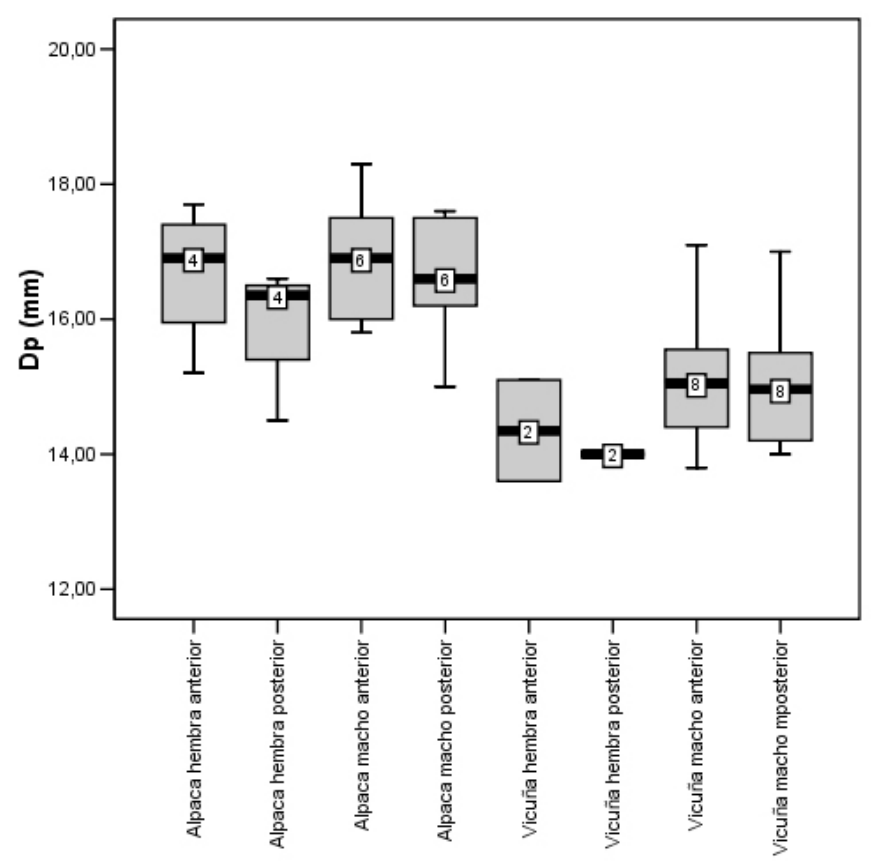

Figura 7b: Medidas de la profundidad máxima (Dp)de las primeras falanges de vicuñas y alpacas actuales separadas por sexo (macho-hembra) y posición (anterior-posterior).

punto de corte que permitiera separar el conjunto de camélidos grandes (guanacos y llamas) y pequeños (vicuñas y alpacas), las medidas fueron llevadas a un gráfico de dispersión (Figura 8). En trabajos anteriores, se había propuesto como punto de corte $18.00 \mathrm{~mm}$ para la medida BFp (Cartajena et al. 2007), la que también se utilizó en este caso y que permite separar claramente ambos grupos.

Al considerar las variables BFp y Dp por separado para el

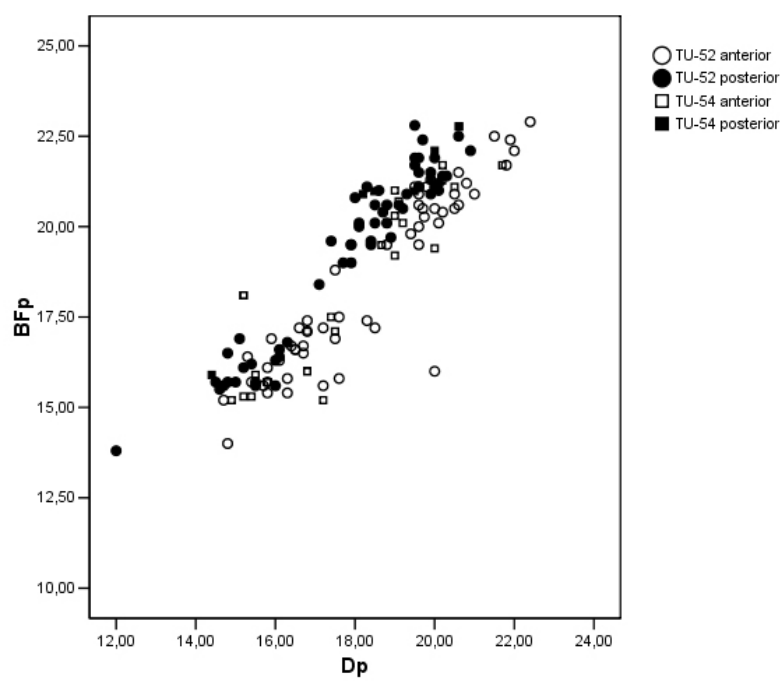

Figura 8: Medidas del ancho máximo de la superficie articular proximal (BFp) y de la profundidad máxima (Dp) de las primeras falanges de los sitios Tulán-52 y 54.

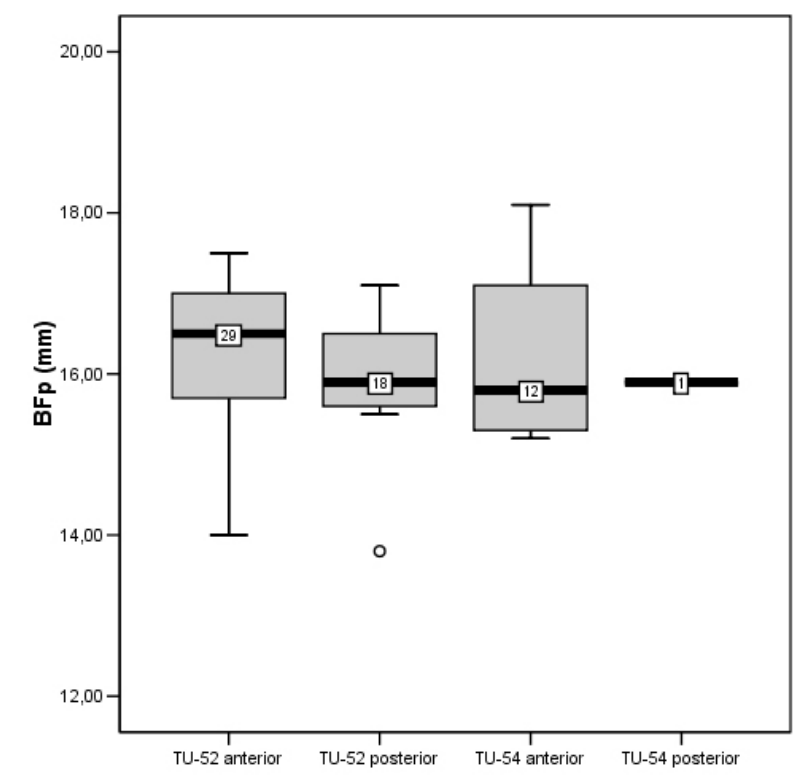

Figura 9: Medidas del ancho máximo de la superficie articular proximal (BFp) de las primeras falanges de los sitios Tulán-52 y 54.

conjunto de camélidos pequeños se puede observar una gran homogeneidad entre ambos sitios (Figuras 9 y 10) .

El ancho máximo de la superficie articular proximal es muy similar en ambos yacimientos ya sea para las falanges anteriores como posteriores. En el caso de Tulán-52 se puede observar una diferencia entre las falanges anteriores y posteriores; sin embargo, esta diferencia no se aprecia en el caso de Tulán-54, aunque debe señalarse que de este sitio sólo se cuenta con una observación para la falange posterior (Figura 9). En el caso de la profundidad máxima se aprecia nuevamente y más claramente la diferencia entre falanges anteriores y posteriores en cada uno de los yacimientos (Figura 10).

Las pruebas de diferencia de medias entre las falanges 
anteriores de ambos conjuntos, ya sea para BFp y Dp no permitieron separar estadísticamente ambos grupos (Anexo 3), denotando gran homogeneidad entre los restos de ambos yacimientos. Esta homogeneidad también puede apreciarse a través del análisis de componentes principales realizado para ambos sitios considerando ambas variables tanto para falanges posteriores como anteriores (Figura 11).

Aunque no existen diferencias estadísticamente significativas que permitan separar los conjuntos empleando BFp y Dp, conviene explorar las diferencias detectadas en los gráficos. Como se puede observar en la Figura 9, BFp es muy similar tanto para las falanges anteriores como posteriores de ambos sitios. Esta situación concuerda con los resultados obtenidos especialmente para las vicuñas actuales donde no se observaba para esta medida una mayor variación entre falanges anteriores y posteriores (Figura 2). Al considerar la medida Dp (Figura 10) sucede algo similar, a lo observado con la medida anterior ya que no presenta grandes diferencias entre anteriores y posteriores (Figura 3). Las similitudes observadas en los conjuntos arqueológicos entre los conjuntos de falanges anteriores en cuanto a la caída de la mediana en el caso de Tulán-54 respecto a Tulán-52 es coincidente con lo observado en la Figura 7, donde las falanges anteriores de vicuñas hembras presentan una mediana más baja en relación a las posteriores de machos. Siguiendo esta línea argumental, sería plausible explicar la diferencia entre las medias de las falanges de ambos conjuntos debido a una mayor representación de individuos femeninos en Tulán-54 en comparación con Tulán-52, donde predominarían los machos.

Las diferencias observables no obedecerían a una

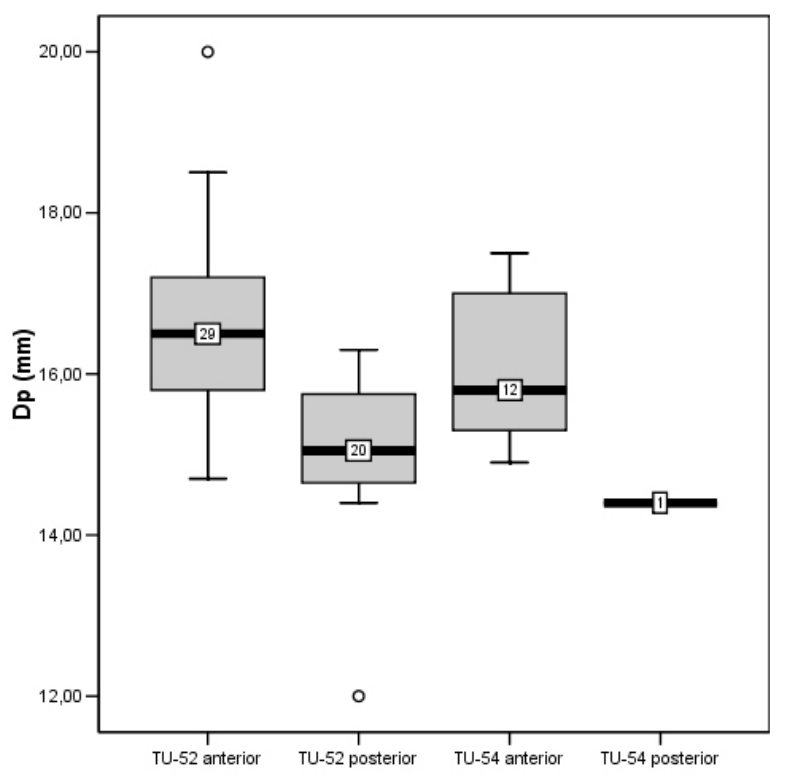

Figura 10: Medidas de la profundidad máxima (Dp) de las primeras falanges de los sitios Tulán-52 y 54 . diferencia en la composición taxonómica, ya que como se observó a través del estudio de colecciones actuales, el ancho máximo de la superficie articular proximal así como la profundidad máxima deberían variar de tal forma que fuesen estadísticamente significativas, lo que no se observa en este caso.

El conjunto de medidas fue comparado con las colecciones actuales de alpacas y vicuñas (Figura 12).

Como se puede observar en el gráfico de dispersión, los restos arqueológicos se encuentran en el rango superior

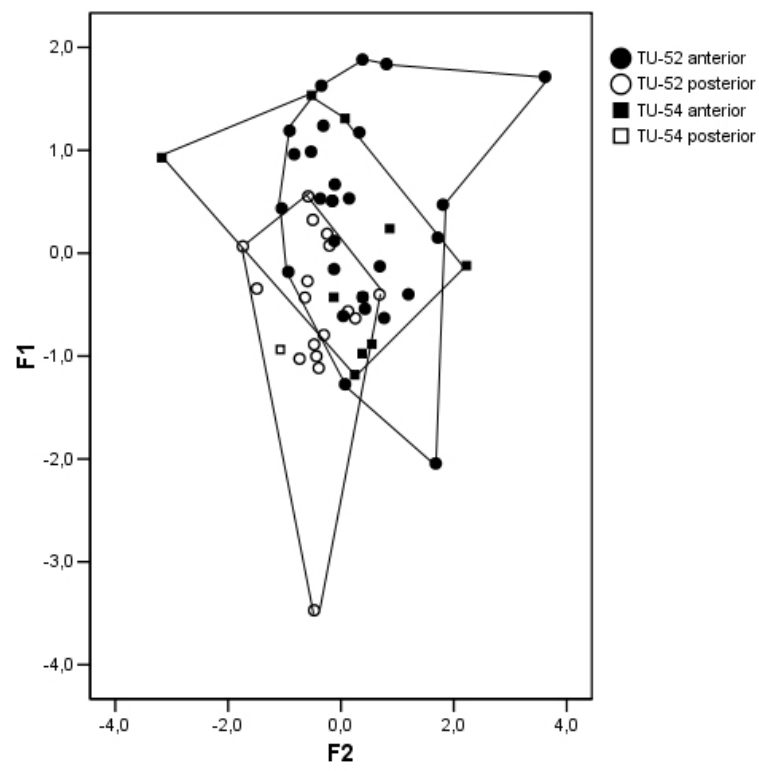

Figura 11: Análisis de componentes principales para dos variables (BFp y DP) para las primeras falanges anteriores y posteriores de los sitios Tulán-52 y 54.

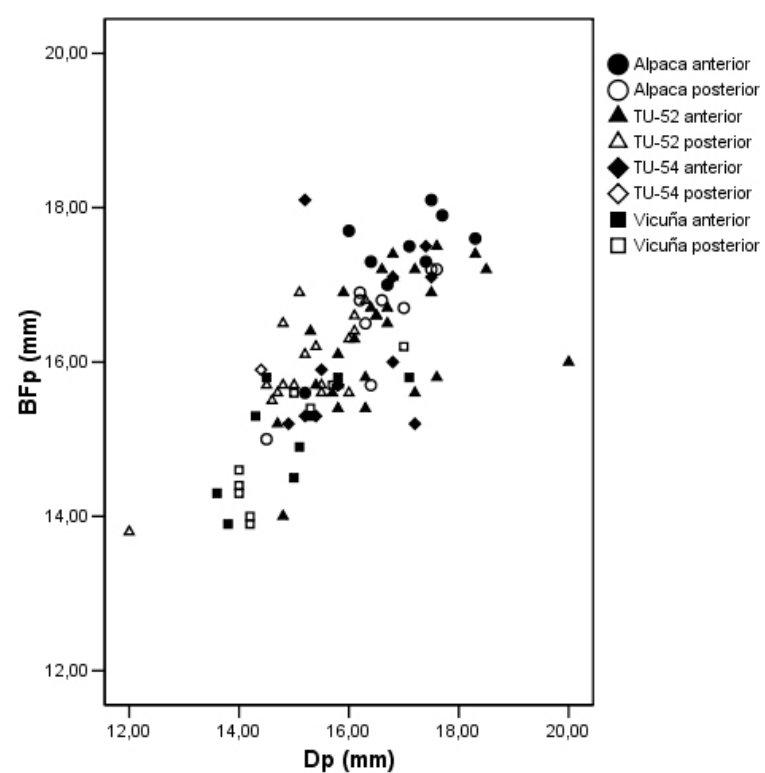

Figura 12: Medidas del ancho máximo de la superficie articular proximal (BFp) y de la profundidad máxima (Dp) de las primeras falanges de los sitios Tulán-52 y 54 comparadas con alpacas y vicuñas actuales. 
de las vicuñas utilizadas como comparación y algunas falanges anteriores de alpacas. Sin embargo, los especimenes recuperados en los sitios arqueológicos no sobrepasan el conjunto de alpacas actuales, con la excepción de una primera falange anterior del sitio Tulán-54.

Si se analizan separadamente las medidas BFp y Dp puede observarse que en el caso de BFp (Figura 13) la mayor parte del conjunto de falanges anteriores de los sitios Tulán-52 y Tulán-54 no alcanzan el tamaño de las alpacas actuales, sucediendo lo mismo con las falanges posteriores. De acuerdo con las pruebas de medias, a

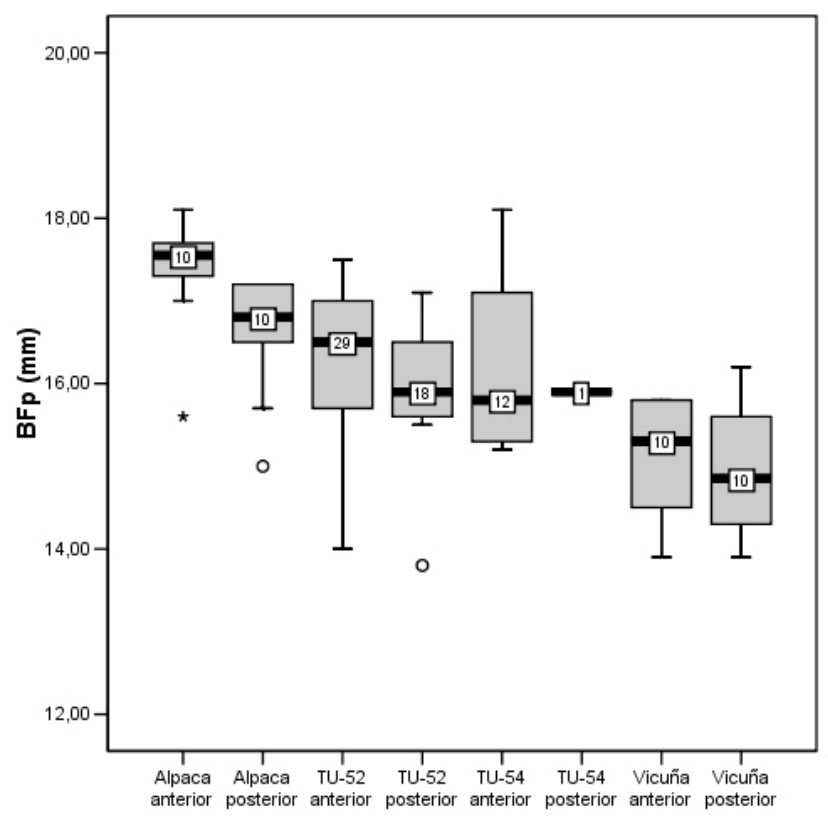

Figura 13: Medidas del ancho máximo de la superficie articular proximal (BFp) de las primeras falanges de los sitios Tulán-52 y 54 comparadas con alpacas y vicuñas actuales.

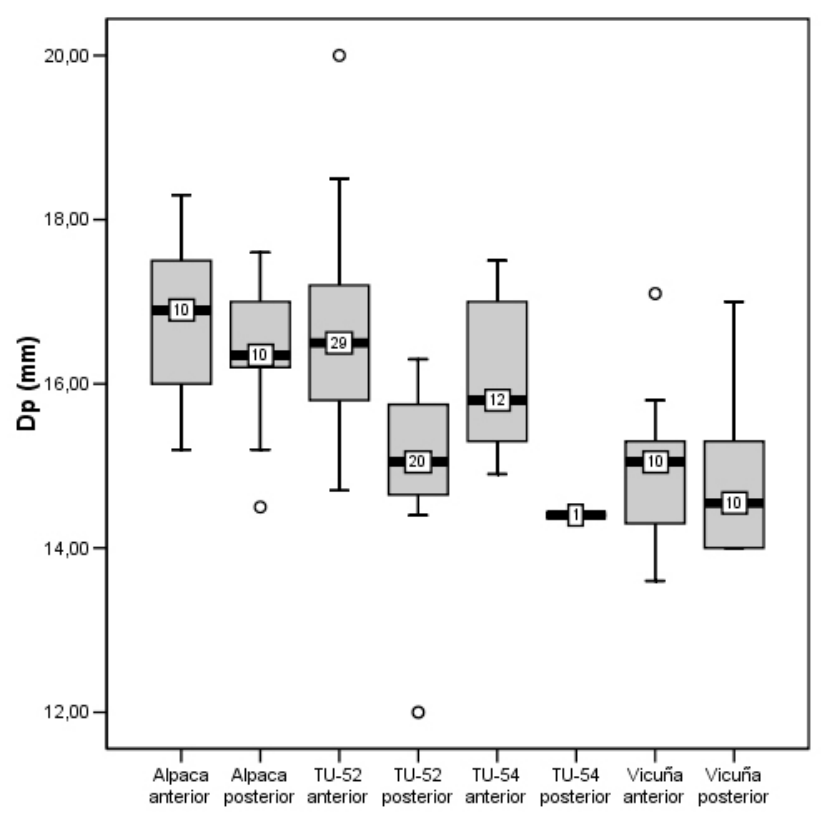

Figura 14: Medidas de la profundidad máxima (Dp) de las primeras falanges de los sitios Tulán-52 y 54 comparadas con alpacas y vicuñas actuales. pesar de la superposición observada, las primeras falanges anteriores tanto de Tulán-52 como de Tulán-54 pueden ser separadas del conjunto de falanges anteriores de alpacas (Figura 14), lo que sugiere que son dos grupos diferentes (Anexo 3). En el caso de la profundidad máxima, esta medida permite separar las falanges posteriores del sitio Tulán-52 del conjunto de las alpacas (Anexo 3).

En el caso de las vicuñas actuales utilizadas como comparación, como se mencionó anteriormente, la mayoría de ellas proviene del área de distribución de Vicugna vicugna mensalis (Wheeler 1995). No obstante, cabe esperar que para el área de estudio se encuentre presente Vicugna vicugna vicugna, subespecie que presenta una alzada mayor (Wheeler 1995). En el caso de las vicuñas del altiplano de Tarapacá, la alzada alcanza incluso tamaños mayores que aquella de las vicuñas más septentrionales (Bonacic 1996). Para nuestro territorio, Glade (1992) reconoce dos conjuntos o razas geograficas, una que habita el altiplano de Tarapacá y otra más austral, que habita el altiplano de las regiones de Antofagasta y Atacama. Nuevos datos provenientes del noroeste argentino muestran la existencia de diferencias significativas con respecto a las vicuñas más septentrionales, ya que se observa un mayor tamaño y masa corporal en las meridionales (Mengoni 2008).

Los antecedentes de vicuñas más tempranas para el área de estudio provienen del sitio Tambillo-1 (8.870 40 A. P. y $8.590 \pm 130$, Núñez et al. 2005) situado en una cota un poco más baja pero que presenta abundantes restos de camélidos pequeños, así como del yacimiento Tulán-68, ubicado en la misma quebrada y datado en 9.290 \pm 100 A. P. (Núñez et al. 2005). Las medidas obtenidas describen un conjunto de individuos que presentan un tamaño mayor que las vicuñas actuales utilizadas como referencia, alcanzando en algunos casos el rango de las alpacas actuales, aunque por el contexto y las fechas se trataría de vicuñas de mayor tamaño (Cartajena 2002, Cartajena et al. 2005). Lo anterior sugiere que las vicuñas que poblaron el área de estudio en la prehistoria habrían alcanzado un tamaño mayor que las de la Puna seca más septentrional. Si bien la mayor parte del conjunto correspondería a vicuñas, en el sitio formativo Tulán-54 se han registrado algunos incisivos con una morfología similar a la descrita para alpaca (Wheeler 1984, Mengoni y Yacobaccio 2006), por lo que no es posible descartar la introducción de algunas alpacas, las que no sería posible diferenciar osteométricamente pero que cabría encontrar en el rango superior de las medidas.

\section{Consideraciones finales}

Los resultados obtenidos muestran, en términos generales, una gran similitud entre los conjuntos del sitio arcaico Tulán-52 y el formativo Tulán-54, lo que denotaría una composición taxonómica similar ya que no se observan las diferencias esperables en el caso que se encontraran representados dos taxones, especialmente al separar falanges anteriores de posteriores. En el caso del conjunto 
formativo, no se observa un aumento en el tamaño de los especimenes que pudiese estar relacionado con la incorporación o una creciente importancia de la alpaca. No obstante, de acuerdo a la morfología dental, no es posible descartar la eventual presencia de algún espécimen.

Los factores que pudiesen estar incidiendo en la variabilidad dentro y entre los conjuntos de camélidos pequeños, se relacionarían con la representación de falanges anteriores y posteriores y con la composición en términos de sexo de los conjuntos.

A pesar de las limitaciones que presentan los estudios osteométricos, estos permiten caracterizar los conjuntos faunísticos en el pasado y contribuir a un mejor conocimiento de la variación regional relacionada con el tamaño de los camélidos. En este caso de estudio, el conjunto de camélidos pequeños atribuido a vicuñas presentaría un tamaño mayor que las vicuñas más septentrionales. Si bien estos resultados pueden variar de región en región, permiten explorar la poco conocida variablidad existente dentro del conjunto de los camélidos pequeños. Los sitios ubicados en quebrada Tulán presentan grandes ventajas para el estudio de restos faunísticos, puesto que además de la continuidad cultural, se ubican en un mismo ambiente. Ambas características permiten un mejor control de aspectos tales como la posible incidencia de variaciones ambientales y su impacto en la variabilidad métrica de los especímenes.

Finalmente, hasta ahora se ha puesto un fuerte énfasis en el registro de camélidos domésticos como indicador de las sociedades formativas. No obstante, la alta presencia de vicuñas en contextos arcaicos y formativos, permite sustentar por una parte, que el proceso de domesticación de la alpaca fue más restringido que el de la llama y que ésta no se encontraría representada tempranamente en la región de estudio, por lo que su introducción habría sido más tardía. Por otra, la fuerte presencia de camélidos silvestres, en este caso de vicuñas, permite denotar la importancia de las prácticas de caza en coexistencia con la tenencia de rebaños durante el Formativo Temprano. La pervivencia de la cacería de vicuñas, especialmente colectiva, se encuentra bien documentada hasta principios del siglo XX en la región (Bowman 1924 en Núñez et al. 2005). En este sentido, el estudio del conjunto de camélidos de tamaño pequeño, no sólo nos permite conocer y caracterizar de mejor forma este grupo de tamaño, sino también comprender la importancia de este conjunto a través del tiempo.

Santiago de Chile, 16 de agosto 2008

\section{Agradecimientos}

Este trabajo se realizó en el marco de los proyectos Fondecyt 1020316 y 1070040 . Mis especiales agradecimientos a A. Izeta por facilitarme sus datos y sus pacientes comentarios y a José Miguel Benavente. De igual forma a las instituciones que me facilitaron el acceso a las colecciones de referencia Staatssammlung für Anthropologie und $\mathrm{Pa}$ läoanatomie y Zoologische Staatssammlung, Muenchen, Alemania y Natural History Museum, Londres.

\section{Bibliografía}

Benavente, M. A. 2005-2006. Análisis lanimétrico de fanéreos de los sitios Tulán 52 y 54. Informe de Avance Proyecto FONDECYT 1020316. Manuscrito

Bonacic, C. 1996. Sustainable use of the vicuñas (Vicugna vicugna) Chile. Tesis de Maestría, Department of Pure and Applied Zoology, University of Reading, Inglaterra.

Bonavía, D. 1996. Los camélidos sudamericanos. Una introducción a su estudio. Travaux de I'Institut Français d'Ètudes Andines 93, Lima.

Cartajena, I. 2002. Los conjuntos arqueofaunísticos del Arcaico Temprano en la Puna de Atacama, Norte de Chile. Tesis doctoral. Freie Universität Berlin. ABESY Vertriebs GmbH, Germany (Impreso en microfilm 2003).

Cartajena, I. e I. Concha. 1997. Una contribución a la determinación taxonómica de la familia Camelidae en sitios formativos del Loa Medio. Estudios Atacameños 14: 71-83.

Cartajena, I., Núñez, L. y M.Grosjean. 2005. Las arqueofaunas del Arcaico Temprano en la vertiente occidental de la Puna de Atacama. Actas XVI Congreso Nacional de Arqueología Chilena, Ediciones Escaparate: 507-517.

Cartajena, I., L. Núñez y M. Grosjean. 2007. Camelid domestication in the western slope of the Puna de Atacama, Northern Chile. Anthropozoologica 42(2): 155-173.

Cartajena, I., A. Benavente, L. Núñez y C. Thomas. 2009. La utilización de los camélidos durante el Formativo Temprano: Una comparación entre la cuenca del Loa Medio y el Salar de Atacama. P. López, I. Cartajena, C. García, y F. Mena (eds.), Zooarqueologia y tafonomia en el confin del mundo. Monografías Arqueológicas 1, Universidad Internacional SEK-Chile, : 181-198.

Driesch, A.von den 1999. A guide to measurement of animal bones from archaeological sites. 5ta edición. Peabody Museum Bulletins 1.

Elkin, D., C. M. Madero, L. G. Mengoni Goñalons, D. E. Olivera y H. D. Yacobaccio. 1991. Avances en el estudio arqueológico de los camélidos en el noroeste argentino. En: Actas de la VII Convención Internacional de Especialistas en Camélidos Sudamericanos (S.S. de Jujuy, Argentina 17-20 de abril de 1991) (no publicado).

Glade, A. 1992. La vicuña. En: Camélidos silvestres sud- 
americanos. Un plan de acción para su conservación. $\mathrm{H}$. Torres (Ed.), International Union for Conservation of Nature and Natural Resources, Gland: 46-48.

Grosjean, M., J. Leeuwe, W. O. van der Knaap, B. Ammann, W. Tanner, B. Messerli, L. Núñez, B. ValeroGarce y H. Veith. 2001. A 22,000 ${ }^{14} \mathrm{C}$ years B.P. sediment and pollen record of climate change of Laguna Miscanti $\left(23^{\circ} \mathrm{S}\right)$, Northern Chile. Global and Planetary Change 28: $35-51$.

Grosjean M., L. Núñez e I. Autor. 2005. Cultural response to climate change in the Atacama Desert. Mike Smith y Paul Hesse (eds.) $23^{\circ}$ South: The Archaeology and Environmental History of the Southern Deserts, National Museum of Australia, Australia; 2005; 144-159.

Herre, W. 1952. Studien über die wilden und domestizierten Tylopoden Südamerikas. Der Zoologische Garten (N.F.) 19 (2/4): 70-98.

Hesse, B. 1982. Archaeological evidence for camelid exploitation in the Chilean Andes. Säugetierkundliche Mitteilungen 30: 201-211.

Izeta, A. D. 2004. Zooarqueología del sur de los valles Calchaquíes. Estudio de conjuntos faunísticos del Período Formativo. Tesis de Doctorado, Facultad de Ciencias Naturales y Museo, Universidad Nacional de La Plata (MS).

Izeta, A. D. 2007. Zooarqueología del sur de los valles Calchaquies (Provincias de Catamarca y Tucumán, (República Argentina). BAR International Series 1612.

Izeta, A. D. y L. I. Cortés. 2006. South American Camelid Paleopathologies: Examples from Loma Alta (Catamarca, Argentina). International Journal of Osteoarchaeology 16: 269-275.

Kadwell, M., M. Fernández, H. F. Stanley, R. Baldi, J. C. Wheeler, R. Rosadio y M. W. Bruford. 2001. Genetic analysis reveals the wild ancestors of the llama and the alpaca. Proceedings Royal Society London 268: 2575-2584.

Kent, J. 1982. The domestication and exploitation of south american camelids: Methods of analysis and their application to circumlacustrine archaeological sites in Bolivia and Peru. Ph. D. thesis, Department of Anthropology, Washington University. University Microfilms International, Ann Arbor, Michigan (impreso en microfilm 1986).

López, C. 2001. Técnicas estadísticas para SPSS. Prentice may, Madrid.

Marín, J. C., B. Zapata, B. A. González, C. Bonacic, J.C. Wheele, C. Casey, M. W. Bruford, R. E. Palma, E. Poulin, M. A. Alliende y A. E. Spotorno. 2007. Sistemática, taxonomía y domesticación de alpacas y llamas: nueva evidencia cromosómica y molecular. Revista Chilena de
Historia Natural 80: 121-140.

Menegaz, A. N., M. C. Salemme y E. Ortiz Jaureguizar. 1988. Una propuesta de sistematización de los caracteres morfométricos de los matapodios y las falanges de Camelidae. N. R. Ratto y A. F. Haber (eds.), De procesos, contextos y otros huesos, Facultad de Filosofía y Letras, U.B.A., Buenos Aires: 53-63.

Menegaz, A. N., F.J. Goin y E. Ortiz Jaureguizar. 1989. Análisis morfológico y morfométrico multivariado de los representantes fósiles y vivientes del género Lama (Artiodactyla, Camelidae). Sus implicancias sistemáticas, biogeográficas, ecológicas y biocronológicas. Ameghiniana 26 (3-4): 153-172.

Mengoni Goñalons, G. L. 2008. Camelids in ancient Andean societies: A review of the zooarchaeological evidence. Quaternary International 185: 59-68.

Mengoni Goñalons, G. L. y H. D.Yacobaccio 2006. The domestication of south american camelids. A view from the South-Central Andes. M. A. Zedar., D. G. Bradley, E. Emshwiller y B. D. Smith (eds), Documenting domestication. New genetic and archaeological paradigms., University of California Press, Berkeley. Los Angeles: 228-244.

Miller, G. R y R. L. Burger. 1995. Our father the Cayman, our dinner the llama: Animal utilization at Chavín de Huantar, Peru. American Antiquity 60(3): 421-458.

Miller, G. R. y A. L. Gill. 1990. Zooarchaeology at Pirincay, a Formative Period Site in Highland Ecuador. Journal of Field Archaeology 17(1): 49-68.

Moore, K. 1989. Hunting and the origin of herding in Peru. Ph. D. thesis, University of Michigan, University Microfilms International, Ann Arbor, Michigan (impreso en microfilm 1989).

Núñez, L. y C. Santoro. 1988. Cazadores de la puna seca y salada del área centro-sur Andina (Norte de Chile). Estudios Atacameños 9: 11-60.

Núñez, L., Grosjean, M. and Autor, I. 2005. Ocupaciones humanas y paleoambientes en la Puna de Atacama. Universidad Católica del Norte-Traxacum, Santiago, Chile.

Núñez, L., I. Autor, C. Carrasco, P. de Souza y M. Grosjean 2006. Emergencia de comunidades pastoralistas formativas en el sureste de la Puna de Atacama. Estudios Atacameños 32: 93-117.

Wheeler, J. C. 1984. La domesticación de la alpaca (Lama pacos L.) y la llama (Lama glama L.) y el desarrollo temprano de la ganadería autóctona en los Andes Centrales. Boletín de Lima 6 (36): 74-84.

Wheeler, J. C. 1991. Origen, evolución y status actual. 
S. Fernández-Baca (ed.), Avances y perspectivas del conocimiento de los camélidos sudamericanos, FAO, Santiago: 11-48.

Wheeler, J. C. 1995. Evolution and present situation of the South American Camelids. Biological Journal of Linnean Society 54: 271-295.

Wing, E. 1972. Utilization of animal resources in the Peruvian Andes. S. Izumi y K. Terada (eds.), Andes 4, Excavations at Kotosh, Peru, 1963 and 1969. University of Tokyo Press: 327 - 350.

Yacobaccio H. D. 2003. Procesos de intensificación y de domesticación de camélidos en los Andes Centro-Sur. III
Congreso Mundial sobre Camélidos. Taller internacional de DECAMA, Potosí, 15-18 octubre 2003. UNEPACA, Bolivia: 211-216.

Yacobaccio, H. D., D. Elkin y D. Olivera 1994. ¿El fin de las sociedades cazadoras? El proceso de domesticación animal en los Andes Centro-sur. J. L. Lanata y L. A. Borrero (eds.), Arqueología de Cazadores-Recolectores: Límites, Casos y Aperturas, Arqueología Contemporánea 5: 23-32.

Yacobaccio, H. D., C. M. Madero, M. P. Malmierca y M. C. Reigadas 1997/1998. Caza, domesticación y pastoreo de camélidos en la Puna Argentina. Relaciones de la Sociedad Argentina de Antropología 22/23: 389-429.

Anexo 1: Medidas del ancho máximo de la superficie articular proximal (BFp) y de la profundidad máxima (Dp) de las primeras falanges (medial) y posterior (medial) en camélidos actuales ( $\mathrm{mm}$ ).

\begin{tabular}{lcccccc}
\hline Especie & Procedencia & Sexo & BFp anterior & Dp anterior & BFp posterior & Dp posterior \\
\hline L. pacos & APSM & $\mathrm{F}$ & 17,5 & 17,1 & 15,7 & 16,4 \\
L. pacos & $\mathrm{APSM}$ & $\mathrm{M}$ & 17,3 & 17,4 & 16,7 & 17,0 \\
L. pacos & $\mathrm{APSM}$ & $\mathrm{M}$ & 18,1 & 17,5 & 17,2 & 17,6 \\
L. pacos & $\mathrm{NHM}^{2}$ & $\mathrm{~F}$ & 17,9 & 17,7 & 16,5 & 16,3 \\
L. pacos & ZSM $^{3}$ & $\mathrm{~F}$ & 15,6 & 15,2 & 15,0 & 14,5 \\
L. pacos & ZSM & $\mathrm{M}$ & 17,7 & 16,0 & 16,9 & 16,2 \\
L. pacos & ZSM & $\mathrm{F}$ & 17,0 & 16,7 & 16,8 & 16,6 \\
L. pacos & ZSM & $\mathrm{M}$ & 17,3 & 16,4 & 16,8 & 16,2 \\
L. pacos & ZSM & $\mathrm{M}$ & 17,6 & 18,3 & 17,2 & 17,5 \\
L. pacos & $\mathrm{DAUCH}$ & $\mathrm{M}$ & 17,6 & 15,8 & 17,2 & 15,0 \\
V. vicugna & $\mathrm{APSM}$ & $\mathrm{M}$ & 15,8 & 17,1 & 16,2 & 17,0 \\
V. vicugna & $\mathrm{APSM}$ & $\mathrm{F}$ & 14,3 & 13,6 & 14,4 & 14,0 \\
V. vicugna & $\mathrm{APSM}$ & $\mathrm{M}$ & 15,8 & 15,8 & 15,4 & 15,3 \\
V. vicugna & $\mathrm{NHM}$ & $\mathrm{M}$ & 13,9 & 13,8 & 13,9 & 14,2 \\
V. vicugna & ZSM & $\mathrm{M}$ & 14,5 & 15,0 & 14,0 & 14,2 \\
V. vicugna & ZSM & $\mathrm{F}$ & 14,9 & 15,1 & 14,3 & 14,0 \\
V. vicugna & ZSM & $\mathrm{M}$ & 15,3 & 14,3 & 14,6 & 14,0 \\
V. vicugna & ZSM & $\mathrm{M}$ & 15,3 & 15,3 & 15,6 & 15,0 \\
V. vicugna & ZSM & $\mathrm{M}$ & 15,8 & 14,5 & 15,7 & 15,7 \\
V. vicugna & $\mathrm{DAUCH}$ & $\mathrm{M}$ & 15,3 & 15,1 & 15,1 & 14,9 \\
\hline
\end{tabular}

\begin{tabular}{lrrrr}
\hline Especie & BFp anterior & Dp anterior & BFp posterior & Dp posterior \\
\hline L. pacos & & & & \\
Media & 17,36 & 16,81 & 16,60 & 16,33 \\
Mediana & 15,55 & 16,90 & 16,80 & 16,35 \\
Desv. Est. & 0,69 & 0,96 & 0,72 & 0,98 \\
Min & 15,10 & 15,20 & 15,00 & 14,50 \\
Max & 18,10 & 18,30 & 17,20 & 17,60 \\
N & 10,00 & 10,00 & 10,00 & 10,00 \\
\hline V. vicugna & & & & \\
Media & 15,09 & 14,96 & 14,92 & 14,83 \\
Mediana & 15,30 & 15,05 & 14,85 & 14,55 \\
Desv. Est. & 0,67 & 1,01 & 0,79 & 0,98 \\
Min & 13,90 & 13,60 & 13,90 & 14,00 \\
Max & 15,80 & 17,10 & 16,20 & 17,00 \\
N & 10,00 & 10,00 & 10,00 & 10,00 \\
\hline
\end{tabular}

1Staatssammlung für Anthropologie und Paläoanatomie (APSM, Muenchen, Alemania)

2 Zoologische Staatssammlung (ZSM, Muenchen, Alemania)

3 Natural History Museum (NHM, Londres)

4 Departamento Antropología, Universidad de Chile (DAUCH, Santiago) 


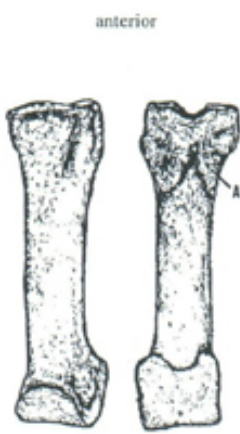

(a)

(b)

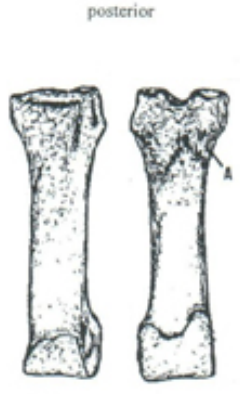

(d) (c)

Primera falange de vicuña

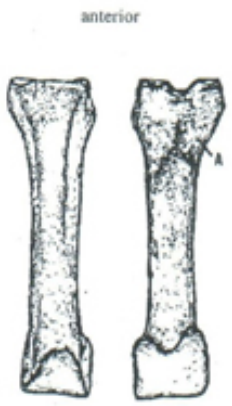

(a)

(b)

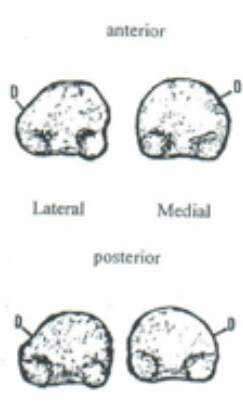

(e)

Anexo 2: Primeras falanges anteriores y posteriores de alpaca y vicuña. a. y c. vista dorsal b. vista palmar d. vista plantar y e. vista proximal (Tomado de Cartajena 2002).

Anexo 3: Pruebas de diferencia de medias

\begin{tabular}{|c|c|c|c|c|c|c|c|c|}
\hline Prueba & Medida & $\mathrm{t}$ & gl & $\begin{array}{c}\text { Sig. } \\
\text { (bilateral) }\end{array}$ & $\begin{array}{l}\text { Diferencia de } \\
\text { medias }\end{array}$ & $\begin{array}{c}\text { Error tip. } \\
\text { Diferencia de } \\
\text { medias }\end{array}$ & Inferior & Superior \\
\hline \multicolumn{9}{|l|}{ Alpaca anterior - posterior } \\
\hline \multirow[b]{3}{*}{ Vicuña anterior - posterior } & BFp & 2,427 & 18 & 0,026 & 0,7650 & 0,31515 & 0,10289 & 1,42711 \\
\hline & $\mathrm{Dp}$ & 1,103 & 18 & 0,284 & 0,4800 & 0,43500 & $-0,43389$ & 1,39389 \\
\hline & $\begin{array}{l}\text { BFp } \\
\text { Dp }\end{array}$ & $\begin{array}{l}0,506 \\
0,285\end{array}$ & $\begin{array}{l}18 \\
18\end{array}$ & $\begin{array}{l}0,619 \\
0,779\end{array}$ & $\begin{array}{l}0,1660 \\
0,1270\end{array}$ & $\begin{array}{l}0,32790 \\
0,44554\end{array}$ & $\begin{array}{l}-0,52290 \\
-0,80904\end{array}$ & $\begin{array}{l}0,85490 \\
1,06304\end{array}$ \\
\hline $\begin{array}{l}\text { Alpaca posterior - Vicuña } \\
\text { anterior }\end{array}$ & $\begin{array}{l}\text { BFp } \\
\text { Dp }\end{array}$ & $\begin{array}{l}4,867 \\
3,071\end{array}$ & $\begin{array}{l}18 \\
18\end{array}$ & $\begin{array}{l}0,000 \\
0,007\end{array}$ & $\begin{array}{l}1,5100 \\
13710\end{array}$ & $\begin{array}{l}0,31023 \\
0,44650\end{array}$ & $\begin{array}{l}0,85822 \\
0,43295\end{array}$ & $\begin{array}{l}2,16178 \\
230905\end{array}$ \\
\hline $\begin{array}{l}\text { Alpaca masculino - } \\
\text { femenino }\end{array}$ & $\begin{array}{l}\text { BFp } \\
\text { Dp }\end{array}$ & $\begin{array}{l}2,146 \\
0,959\end{array}$ & $\begin{array}{l}18 \\
18\end{array}$ & $\begin{array}{l}0,062 \\
0,350\end{array}$ & $\begin{array}{l}0,7992 \\
0,4292\end{array}$ & $\begin{array}{l}0,37233 \\
0,44745\end{array}$ & $\begin{array}{l}-0,04999 \\
-0,51088\end{array}$ & $\begin{array}{l}1,64832 \\
1,36921\end{array}$ \\
\hline $\begin{array}{l}\text { Vicuña masculino - } \\
\text { femenino }\end{array}$ & $\begin{array}{l}\text { BFp } \\
\text { Dp }\end{array}$ & $\begin{array}{l}1,730 \\
1,745 \\
\end{array}$ & $\begin{array}{l}18 \\
18 \\
\end{array}$ & $\begin{array}{l}0,101 \\
0,098 \\
\end{array}$ & $\begin{array}{l}0,6612 \\
0,9006\end{array}$ & $\begin{array}{l}0,38223 \\
0,51624 \\
\end{array}$ & $\begin{array}{l}-0,14179 \\
-0,18395 \\
\end{array}$ & $\begin{array}{l}1,46429 \\
1,98520\end{array}$ \\
\hline $\begin{array}{l}\text { Alpaca femenino - Vicuña } \\
\text { masculino }\end{array}$ & $\begin{array}{l}\text { BFp } \\
\text { Dp }\end{array}$ & $\begin{array}{l}3,793 \\
2,895 \\
\end{array}$ & $\begin{array}{l}22 \\
22 \\
\end{array}$ & $\begin{array}{l}0,001 \\
0,008 \\
\end{array}$ & $\begin{array}{l}1,3638 \\
1,2369 \\
\end{array}$ & $\begin{array}{l}0,35954 \\
0,42718 \\
\end{array}$ & $\begin{array}{l}0,61810 \\
0,35095 \\
\end{array}$ & $\begin{array}{l}2,10940 \\
2,12280 \\
\end{array}$ \\
\hline $\begin{array}{l}\text { TU-54 anterior - TU-52 } \\
\text { anterior }\end{array}$ & $\begin{array}{l}\text { BFp } \\
\text { Dp }\end{array}$ & $\begin{array}{l}-0,550 \\
-1,302 \\
\end{array}$ & $\begin{array}{l}39 \\
39 \\
\end{array}$ & $\begin{array}{l}0,585 \\
0,201\end{array}$ & $\begin{array}{l}-0,1664 \\
-0,4819 \\
\end{array}$ & $\begin{array}{l}0,30231 \\
0,37010 \\
\end{array}$ & $\begin{array}{l}-0,77786 \\
-1,23049 \\
\end{array}$ & $\begin{array}{l}0,44510 \\
0,26670\end{array}$ \\
\hline $\begin{array}{l}\text { TU-54 posterior - TU-52 } \\
\text { posterior }\end{array}$ & $\begin{array}{l}\text { BFp } \\
\text { Dp }\end{array}$ & $\begin{array}{l}-0,116 \\
-0,693 \\
\end{array}$ & $\begin{array}{l}17 \\
19 \\
\end{array}$ & $\begin{array}{l}0,909 \\
0,497 \\
\end{array}$ & $\begin{array}{l}-0,0889 \\
-0,6700 \\
\end{array}$ & $\begin{array}{l}0,76794 \\
0,96704 \\
\end{array}$ & $\begin{array}{l}-1,70910 \\
-2,69403 \\
\end{array}$ & $\begin{array}{l}1,53132 \\
1,35403 \\
\end{array}$ \\
\hline $\begin{array}{l}\text { TU-52 anterior - Alpaca } \\
\text { anterior }\end{array}$ & $\begin{array}{l}\text { BFp } \\
\text { Dp }\end{array}$ & $\begin{array}{l}-3,228 \\
-0,759 \\
\end{array}$ & $\begin{array}{l}36 \\
36 \\
\end{array}$ & $\begin{array}{l}0,003 \\
0,453 \\
\end{array}$ & $\begin{array}{l}-0,9920 \\
-0,3153 \\
\end{array}$ & $\begin{array}{l}0,30730 \\
0,41534 \\
\end{array}$ & $\begin{array}{l}-1,61519 \\
-1,15767 \\
\end{array}$ & $\begin{array}{r}-0,36871 \\
0,52702 \\
\end{array}$ \\
\hline $\begin{array}{l}\text { TU-54 anterior - Alpaca } \\
\text { anterior }\end{array}$ & $\begin{array}{l}\text { BFp } \\
\text { Dp }\end{array}$ & $\begin{array}{l}-2,916 \\
-1,902 \\
\end{array}$ & $\begin{array}{l}19 \\
19 \\
\end{array}$ & $\begin{array}{l}0,009 \\
0,072 \\
\end{array}$ & $\begin{array}{l}-1,1583 \\
-0,7972 \\
\end{array}$ & $\begin{array}{l}0,39728 \\
0,41904 \\
\end{array}$ & $\begin{array}{l}-1,98985 \\
-1,67428 \\
\end{array}$ & $\begin{array}{r}-0,32681 \\
0,07984 \\
\end{array}$ \\
\hline $\begin{array}{l}\text { TU-52 posterior - Alpaca } \\
\text { posterior }\end{array}$ & $\begin{array}{l}\text { BFp } \\
\text { Dp }\end{array}$ & $\begin{array}{l}-1,799 \\
-3,750 \\
\end{array}$ & $\begin{array}{l}25 \\
27\end{array}$ & $\begin{array}{l}0,084 \\
0,001\end{array}$ & $\begin{array}{l}-0,5444 \\
-1,4078\end{array}$ & $\begin{array}{l}0,30263 \\
0,37540\end{array}$ & $\begin{array}{l}-1,16772 \\
-2,17804\end{array}$ & $\begin{array}{r}0,07883 \\
-0,63751\end{array}$ \\
\hline
\end{tabular}

